Journal of Thermal Engineering, Vol. 6, No. 3, pp. 405-421, April, 2020

Yildiz Technical University Press, Istanbul, Turkey

\title{
MULTIFLUID DESCRIPTION OF RAREFIED GAS MIXTURE FLOWS
}

\author{
Iman Zahmatkesh ${ }^{1 *}$, Homayoun Emdad², Mohammad M. Alishahi²
}

\begin{abstract}
In the present contribution attention is focused to extend the application of multifluid descriptions to rarefied conditions for the first time. To this aim, a multifluid Maxwell model and a multifluid Smoluchowski model are proposed for near wall behavior of the constituents of a rarefied gas mixture. Afterwards, multifluid balance equations in conjunction with these boundary conditions are solved for some slip flows of binary gas mixtures between parallel plates. The corresponding results are compared with those of a previously developed Navier-Stokes solver. Inspection of the results indicates that while the Navier-Stokes equations may lose their accuracy under high rarefaction, non-equilibrium features are properly captured by developed multifluid description. This successful method is thereafter utilized to discuss the consequences of velocity-slip, the tangential-momentum-accommodation coefficient, and mass disparity of the mixture constituents on the degree of non-equilibrium between the constituents of the gas mixtures between parallel plates.
\end{abstract}

\section{Keywords: Gas Mixture, Kinetic Theory, Rarefied Flow, Multifluid Model, Navier-Stokes Equations INTRODUCTION}

Rarefied gas flows are generally encountered in small geometries, such as micro-sized devices, and in low pressure applications, such as high-altitude aerodynamics. Previous experimental and numerical evidences have demonstrated that, fluid mechanics and heat transfer in such flows are distinct from the continuum counterparts that is attributed to insufficient collision frequencies in the rarefied situations [1]. In spite of the fact that gas mixtures appear in practice more often than a single gas, rarefied gas mixture flows have not been studied extensively in the past.

Mixing of two parallel gas streams in a microchannel was investigated by Yan and Farouk [2], Wang and Li [3], and Hosseinalipour et al. [4] adopting the DSMC method. A DSMC simulation of rarefied flows of binary gas mixtures around five special nose cone shapes was also undertaken by Amini et al. [5]. Moreover, Vargas et al. [6] employed the DSMC method for the simulation of transient behavior of rarefied binary gas mixtures confined between two coaxial cylinders with sudden change of the surface temperatures. Meanwhile, Naris et al. [7,8] and Kosuge and Takata [9] employed the McCormack kinetic model [10] for the analysis of rarefied binary gas mixture flows between parallel plates driven by small pressure, temperature, and concentration gradients. A McCormack simulation of gas mixture flows in triangular and trapezoidal microchannels was undertaken by Szalmas and Valougeorgis [11]. Polikarpov et al. [12] adopted the McCormack model to discuss the transient heat transfer occurring in a rarefied gas mixture confined between parallel plates due to sudden small change of the wall temperatures. Furthermore, Ho et al. [13] examined the accuracy of the McCormack model by comparing its results for the Couette and Fourier flows of gas mixtures with those obtained from the linearized Boltzmann equation. Tantos and Valougeorgis [14] solved the Kosuge kinetic model [15] for the problem of heat transfer in rarefied gas mixtures between two parallel plates. An extension of this work for the Couette flows was presented by Tantos [15]. More recently, Yamaguchi et al. [17] measured the conductive heat transfer through rarefied binary gas mixtures of helium and argon.

Zahmatkesh et al. [18] have derived new velocity-slip and temperature-jump boundary conditions for a whole rarefied gas mixture and solved the Navier-Stokes equations for a $\mathrm{He}-\mathrm{Ne}$ flow in a microchannel. This approach was thereafter adopted for the analysis of low pressure gas mixture flows in a converging-diverging

This paper was recommended for publication in revised form by Regional Editor Ozgen Acikgoz

${ }^{1}$ Department of Mechanical Engineering, Mashhad Branch, Islamic Azad University, Mashhad, Iran

${ }^{2}$ School of Mechanical Engineering, Shiraz University, Shiraz, Iran

${ }^{\star}$ E-mail address: Zahmatkesh5310@mshdiau.ac.ir

Orcid id: 0000-0003-2573-6789;0000-0001-5870-384X; 0000-0002-1286-6691

Manuscript Received 27 June 2018, Accepted 23 August 2018 
nozzle, wall-cooling of a nozzle with blowing of a foreign gas under rarefied condition, and parallel mixing in a microchannel [19].

In spite of previous advancements in the analysis of rarefied gas mixtures flows, multifluid models have not been employed for the description of these flow fields yet. This approach has a wide range of application for the simulation of gas-gas, gas-liquid, and solid-liquid mixture problems [20-24].

In the present contribution, a multifluid model with previous success in the simulation of monoatomic gases in the continuum flow regime [25-28] is applied to some slip flows of binary gas mixtures between parallel plates. This is attained by the application of separate velocity-slip and temperature-jump boundary conditions for each of the constituents. In this regard, all of the boundary conditions for the single-component rarefied flows will be applicable. Such an approach, however, leads to the loss of crucial physics in the simulation of near wall behavior since it ignores the mutual influence of the constituents onto each other. Moreover, the effect of molecular interaction model on the slipping species remains unexplored. To overcome the aforementioned shortcomings, in this contribution, a multifluid Maxwell model and a multifluid Smoluchowski model are proposed as extensions of the Maxwell [29] and Smoluchowski [30] boundary conditions for separate component species in multifluid descriptions.

\section{THE RAREFIED BOUNDARY CONDITIONS The Multifluid Maxwell Model}

The generalized version of the Maxwell's slip condition takes the form of:

$$
u_{s}-u_{w}=\frac{(2-\sigma)}{\sigma} \frac{\lambda}{\mu} \boldsymbol{\tau}_{t, w}-\frac{3}{4} \frac{(\gamma-1)}{\gamma} \frac{\operatorname{Pr}}{p} \boldsymbol{q}_{t, w}
$$

Here, $u_{s}$ and $u_{w}$ are the slip and wall velocities, $p$ is pressure, $\mu$ and $\gamma$ are the fluid viscosity and specific heat ratio, $\lambda$ is the mean-free-path, and $\operatorname{Pr}$ is the Prandtl number. Moreover, $\sigma$ stands for the tangential-momentumaccommodation coefficient. Meanwhile, $\boldsymbol{\tau}_{t, w}$ and $\boldsymbol{q}_{t, w}$ represent the tangential shear stress and heat flux at the wall, respectively. It is worth noting that Maxwell [29] initially derived his slip law in this form and then simplified it in terms of velocity and temperature gradients. However, due to the relative simplicity of the latter form, it is generally remembered as the Maxwell's main theoretical result.

After applying the above expression to the constituent $\alpha$ of a gas mixture, one arrives at the following expression as a multifluid Maxwell model:

$$
u_{s}^{\alpha}-u_{w}=\frac{\left(2-\sigma_{\alpha}\right)}{\sigma_{\alpha}} \frac{\lambda_{\alpha}}{\eta_{\alpha}} \boldsymbol{\tau}_{t, w}^{\alpha}-\frac{3}{4} \frac{\left(\gamma_{\alpha}-1\right)}{\gamma_{\alpha}} \frac{\operatorname{Pr}}{p_{\alpha}} \boldsymbol{q}_{t, w}^{\alpha}
$$

Here, $\eta_{\alpha}$ is the viscosity coefficient of the component species $\alpha$ in the mixture with $v$ constituents and can be calculated as [25]:

$$
\eta_{\alpha}=\sum_{\gamma=1}^{v} \eta_{\alpha \gamma}
$$

Since no commitment was made to the form of shear stress tensor or heat flux vector, constitutive relations for $5 v$-moment theory [25] is invoked here that leads to:

$$
\boldsymbol{\tau}_{t, w}^{\alpha}=\sum_{\gamma=1}^{v} \eta_{\alpha \gamma}\left(\frac{\partial u_{\gamma}}{\partial n}\right)_{s}
$$




$$
\boldsymbol{q}_{t, w}^{\alpha}=-\sum_{\gamma=1}^{v} \lambda_{\alpha \gamma}\left(\frac{\partial T_{\gamma}}{\partial x}\right)_{s}-\sum_{\gamma=1}^{\nu-1} M_{\alpha \gamma}\left(u_{s}^{\gamma}-u_{s}^{v}\right)
$$

Here, $x$ and $n$ are the streamwise and normal coordinates, respectively, while $u$ and $v$ are the corresponding velocity components. Moreover, $u_{s}^{v}$ is the slip velocity of the component species $v$. Meanwhile, the coefficients $\eta_{\alpha \gamma}$, $\lambda_{\alpha \gamma}$, and $M_{\alpha \gamma}$ are the transport coefficients associated with viscous, thermal, and thermal-diffusion effects and can be described in terms of the Chapman-Cowling collision integrals [25].

Inspection of equations (2)-(5) indicates that in the proposed slip law, the mutual influence of the constituents onto each other is calculated. Moreover, the effect of molecular interaction model is included therein through the transport coefficients. Additionally, the proposed condition computes the contribution of mass diffusion in energy transport within slipping species.

\section{The Multifluid Smoluchowski Model}

Using the same procedure as the slip condition, one may arrive at a multifluid Smoluchowski model for the constituent $\alpha$ of the gas mixture as:

$$
T_{g}^{\alpha}-T_{w}=-\frac{2-\sigma_{T, \alpha}}{\sigma_{T, \alpha}} \frac{2 \gamma_{\alpha}}{\gamma_{\alpha}+1} \frac{\lambda_{\alpha}}{\eta_{\alpha} c_{p, \alpha}} \boldsymbol{q}_{n, w}^{\alpha}
$$

Here, $T_{w}$ is the wall temperature, $T_{g}^{\alpha}$ is the gas temperature, $c_{p, \alpha}$ is the constant pressure specific heat, $\gamma_{\alpha}$ is the ratio of the specific heats, $\lambda_{\alpha}$ is the mean-free-path, and $\sigma_{T, \alpha}$ is the thermal-accommodation coefficient, all for the constituent $\alpha$. Meanwhile, $\boldsymbol{q}_{n, w}^{\alpha}$ represents the normal heat flux at the wall. Adopting the $5 v$-moment theory [25], this flux can be expressed as:

$$
\boldsymbol{q}_{n, w}^{\alpha}=-\sum_{\beta=1}^{v} \lambda_{\alpha \beta}\left(\frac{\partial T_{\beta}}{\partial n}\right)_{s}
$$

\section{MULTIFLUID BALANCE EQUATIONS}

The employed multifluid model consists of the following balance equations for the constituent of a binary gas mixture (i.e., $\alpha$ and $\beta$ ) [25]:

$$
\begin{aligned}
& \frac{\partial \boldsymbol{Q}_{\alpha}}{\partial t}+\frac{\partial \boldsymbol{F}_{\alpha}}{\partial x}+\frac{\partial \boldsymbol{G}_{\alpha}}{\partial y}=\frac{\partial \boldsymbol{F}_{v}^{\alpha}}{\partial x}+\frac{\partial \boldsymbol{G}_{v}^{\alpha}}{\partial y}+\boldsymbol{H}_{\alpha} \\
& \frac{\partial \boldsymbol{Q}_{\beta}}{\partial t}+\frac{\partial \boldsymbol{F}_{\beta}}{\partial x}+\frac{\partial \boldsymbol{G}_{\beta}}{\partial y}=\frac{\partial \boldsymbol{F}_{v}^{\beta}}{\partial x}+\frac{\partial \boldsymbol{G}_{v}^{\beta}}{\partial y}+\boldsymbol{H}_{\beta}
\end{aligned}
$$

where:

$$
\boldsymbol{Q}_{\alpha}=\left\{\begin{array}{c}
\rho_{\alpha} \\
\rho_{\alpha} u_{\alpha} \\
\rho_{\alpha} v_{\alpha} \\
E_{\alpha}
\end{array}\right\}, \boldsymbol{F}_{\alpha}=\left\{\begin{array}{c}
\rho_{\alpha} u_{\alpha} \\
\rho_{\alpha} u_{\alpha}^{2}+p_{\alpha} \\
\rho_{\alpha} u_{\alpha} v_{\alpha} \\
\left(E_{\alpha}+p_{\alpha}\right) u_{\alpha}
\end{array}\right\}, \boldsymbol{G}_{\alpha}=\left\{\begin{array}{c}
\rho_{\alpha} v_{\alpha} \\
\rho_{\alpha} u_{\alpha} v_{\alpha} \\
\rho_{\alpha} v_{\alpha}^{2}+p_{\alpha} \\
\left(E_{\alpha}+p_{\alpha}\right) v_{\alpha}
\end{array}\right\}
$$


Journal of Thermal Engineering, Research Article, Vol. 6, No. 3, pp. 405-421, April, 2020

$$
\begin{gathered}
\boldsymbol{F}_{v}^{\alpha}=\left\{\begin{array}{c}
0 \\
\tau_{x x}^{\alpha} \\
\tau_{x y}^{\alpha} \\
u_{\alpha} \tau_{x x}^{\alpha}+v_{\alpha} \tau_{x y}^{\alpha}-q_{x}^{\alpha}
\end{array}\right\}, \boldsymbol{G}_{v}^{\alpha}=\left\{\begin{array}{c}
0 \\
\tau_{y x}^{\alpha} \\
\tau_{y y}^{\alpha} \\
u_{\alpha} \tau_{y x}^{\alpha}+v_{\alpha} \tau_{y y}^{\alpha}-q_{y}^{\alpha}
\end{array}\right\} \\
\boldsymbol{G}_{v}^{\alpha}=\left\{\begin{array}{c}
0 \\
\rho_{\alpha} v_{\alpha \beta}\left(\tilde{u}_{\alpha}-u_{\alpha}\right) \\
\rho_{\alpha} v_{\alpha \beta}\left(\tilde{v}_{\alpha}-v_{\alpha}\right) \\
v_{\alpha \beta}\left(\tilde{E}_{\alpha}-E_{\alpha}\right)
\end{array}\right\}
\end{gathered}
$$

and:

$$
\begin{gathered}
\boldsymbol{Q}_{\beta}=\left\{\begin{array}{c}
\rho_{\beta} \\
\rho_{\beta} u_{\beta} \\
\rho_{\beta} v_{\beta} \\
E_{\beta}
\end{array}\right\}, \boldsymbol{F}_{\beta}=\left\{\begin{array}{c}
\rho_{\beta} u_{\beta} \\
\rho_{\beta} u_{\beta}^{2}+p_{\beta} \\
\rho_{\beta} u_{\beta} v_{\beta} \\
\left(E_{\beta}+p_{\beta}\right) u_{\beta}
\end{array}\right\}, \boldsymbol{G}_{\beta}=\left\{\begin{array}{c}
\rho_{\beta} v_{\beta} \\
\rho_{\beta} u_{\beta} v_{\beta} \\
\rho_{\beta} v_{\beta}^{2}+p_{\beta} \\
\left(E_{\beta}+p_{\beta}\right) v_{\beta}
\end{array}\right\} \\
\boldsymbol{F}_{v}^{\beta}=\left\{\begin{array}{c}
0 \\
\tau_{y x}^{\beta} \\
\tau_{x x}^{\beta} \\
\tau_{y y}^{\beta} \\
u_{\beta} \tau_{x x}^{\beta}+v_{\beta} \tau_{x y}^{\beta}-q_{x}^{\beta}
\end{array}\right\}, \boldsymbol{G}_{v}^{\beta}=\left\{\begin{array}{c}
\tau_{\beta} \\
u_{y x} \tau_{y y}^{\beta}-q_{y}^{\beta}
\end{array}\right\} \\
\boldsymbol{G}_{v}^{\beta}=\left\{\begin{array}{c}
0 \\
\rho_{\beta} v_{\beta \alpha}\left(\tilde{u}_{\beta}-u_{\beta}\right) \\
\rho_{\beta} v_{\beta \alpha}\left(\tilde{v}_{\beta}-v_{\beta}\right) \\
v_{\beta \alpha}\left(\tilde{E}_{\beta}-E_{\beta}\right)
\end{array}\right\}
\end{gathered}
$$

Here, $u_{\alpha}$ and $v_{\alpha}$ are the components of the velocity vector $\left(\mathbf{v}_{\alpha}\right), q_{x}^{\alpha}$ and $q_{y}^{\alpha}$ are the components of the heat flux vector, $\tau_{x x}, \tau_{x y}, \tau_{y x}$, and $\tau_{y y}$ are the components of the viscous stress tensor, $\rho_{\alpha}$ is density, and $E_{\alpha}$ is the total energy per unit volume, all for the constituent $\alpha$. Moreover, $v_{\alpha \beta}$ and $v_{\beta \alpha}$ are the frequencies of cross-collision between $\alpha$ and $\beta$ which are obtained from:

$$
\begin{gathered}
v_{\alpha \beta}=\frac{16}{3} \frac{\rho_{\beta}}{m_{\beta}} \mathbf{\Omega}_{\alpha \beta}^{(1,1)} \\
v_{\beta \alpha}=\frac{\rho_{\alpha}}{\rho_{\beta}} \frac{m_{\beta}}{m_{\alpha}} v_{\alpha \beta}
\end{gathered}
$$

Here, $m_{\alpha}$ and $m_{\beta}$ are the molecular masses and $\boldsymbol{\Omega}_{\alpha \beta}^{(i, j)}$ denotes the Chapman-Cowling collision integrals. The integrals for a Lennard-Jones 12-6 interaction can be found elsewhere [25].

The collision parameters in the balance equations are calculated from the following kinetic relations:

$$
\tilde{\mathbf{v}}_{\alpha}=\tilde{\mathbf{v}}_{\beta}=\frac{m_{\alpha} \mathbf{v}_{\alpha}+m_{\beta} \mathbf{v}_{\beta}}{m_{\alpha}+m_{\beta}}
$$




$$
\begin{gathered}
\tilde{E}_{\alpha}=\rho_{\alpha}\left(\frac{R_{\alpha} \tilde{T}}{\gamma_{\alpha}-1}+\frac{\tilde{\mathbf{v}}_{\alpha}^{2}}{2}\right) \\
\tilde{E}_{\beta}=\rho_{\beta}\left(\frac{R_{\beta} \tilde{T}}{\gamma_{\beta}-1}+\frac{\tilde{\mathbf{v}}_{\beta}^{2}}{2}\right) \\
\tilde{T}_{\alpha}=T_{\alpha}+2 \frac{m_{\alpha} m_{\beta}}{\left(m_{\alpha}+m_{\beta}\right)^{2}}\left(\left(T_{\beta}-T_{\alpha}\right)+\frac{m_{\beta}}{6 k}\left(\mathbf{v}_{\alpha}-\mathbf{v}_{\beta}\right)^{2}\right) \\
\tilde{T}_{\beta}=T_{\beta}+2 \frac{m_{\alpha} m_{\beta}}{\left(m_{\alpha}+m_{\beta}\right)^{2}}\left(\left(T_{\alpha}-T_{\beta}\right)+\frac{m_{\beta}}{6 k}\left(\mathbf{v}_{\alpha}-\mathbf{v}_{\beta}\right)^{2}\right)
\end{gathered}
$$

with $R_{\alpha}$ being the gas constant.

The constitutive relations for viscous stress tensor and heat flux vector are:

$$
\begin{aligned}
\tau_{i j}^{\alpha} & =\sum_{\gamma=1}^{2} \eta_{\alpha \gamma}\left(\frac{\partial v_{i}^{\gamma}}{\partial x_{j}}+\frac{\partial v_{j}^{\gamma}}{\partial x_{i}}-\frac{2}{3} \frac{\partial v_{k}^{\gamma}}{\partial x_{k}} \delta_{i j}\right) \\
\tau_{i j}^{\beta} & =\sum_{\gamma=1}^{2} \eta_{\beta \gamma}\left(\frac{\partial v_{i}^{\gamma}}{\partial x_{j}}+\frac{\partial v_{j}^{\gamma}}{\partial x_{i}}-\frac{2}{3} \frac{\partial v_{k}^{\gamma}}{\partial x_{k}} \delta_{i j}\right) \\
q_{i}^{\alpha} & =-\sum_{\gamma=1}^{2} \lambda_{\alpha \gamma} \frac{\partial T_{\gamma}}{\partial x_{i}}-M_{\alpha \alpha}\left(v_{i}^{\alpha}-v_{i}^{\beta}\right) \\
q_{i}^{\beta} & =-\sum_{\gamma=1}^{2} \lambda_{\beta \gamma} \frac{\partial T_{\gamma}}{\partial x_{i}}-M_{\beta \alpha}\left(v_{i}^{\alpha}-v_{i}^{\beta}\right)
\end{aligned}
$$

The both sets of the balance equations in conjunction with the proposed velocity-slip and temperature-jump boundary conditions are solved invoking explicit flux differencing of the Roe's scheme [31]. In this method, the inviscid terms are calculated based on the evaluation of eignvalues and eigenvectors of the Euler's equations. The viscous terms are also discretized using a central differencing. More details are presented in [26] and are not repeated here for the sake of brevity. From the converged solution, the mass density, velocity, and temperature of the whole mixture are obtained as:

$$
\begin{gathered}
\rho=\rho_{\alpha}+\rho_{\beta} \\
\mathbf{v}=\frac{\rho_{\alpha} \mathbf{v}_{\alpha}+\rho_{\beta} \mathbf{v}_{\beta}}{\rho_{\alpha}+\rho_{\beta}} \\
T=\frac{T_{\alpha} \rho_{\alpha} / m_{\alpha}+T_{\beta} \rho_{\beta} / m_{\beta}}{\rho_{\alpha} / m_{\alpha}+\rho_{\beta} / m_{\beta}}
\end{gathered}
$$

\section{SIMULATION RESULTS}

In this section, simulation results are presented for rarefied gas mixture flows between parallel plates. Helium and xenon are firstly concerned as the mixture constituents. It must be noted that simultaneous use of the proposed velocity-slip and temperature-jump boundary conditions for both sets of the balance equations is beyond the current computational analysis. Consequently, during the present simulations, the walls are concerned adiabatic 
and thereby just the velocity-slip condition is applied with the balance equations. The computations are undertaken for three cases of the gas rarefaction in the slip flow regime. Details of the Case I are:

Problem geometry

Inlet conditions

Exit condition

Wall conditions

Distance between the plates: $2 H=1 \mathrm{~mm}$,

Aspect ratio of the channel: $L / 2 H=10$.

$$
\begin{gathered}
\text { Stagnation pressure: } p_{t, i}=12 \mathrm{kPa}, \\
\text { Stagnation temperature: } T_{t, i}=250 \mathrm{~K}, \\
\text { Mixture concentration: } c_{i}=\frac{\rho_{H e}}{\rho_{H e}+\rho_{X e}}=0.5 .
\end{gathered}
$$

Pressure: $p_{e}=6 \mathrm{kPa}$.

Thermal condition: Adiabatic,

Tangential-momentum-accommodation coefficient: $\sigma=0.85$.

Here, the overall Knudsen number of the whole mixture [18] does not exceed 0.001 over the entire flow field and thereby, the Case I corresponds to the lower limit of the gas rarefaction in the slip flow regime.

In the Case II, rarefaction is increased by prescribing $2 H=100 \mu \mathrm{m}$. Other details are, however, similar to the case I. This makes the maximum value of the overall Knudsen number about 0.01 . Nevertheless, the Case III represents a flow in the upper limit of the gas rarefaction in slip flow regime (i.e., $K n \approx 0.1$ ). This is achieved by assigning $2 H=10 \mu \mathrm{m}$.

The problems are solved employing $4500(100 \times 45)$ nodes based on a grid refinement study. Results provide details of the flow fields for each component species separately. For instance, velocity contours of helium and xenon for the three cases of the gas rarefaction are shown in Figures 1-3. Evidently, the flow is accelerated in accordance with density drop brought about by the decrease in pressure in a way that is consistent with the mass conservation principle. It must be mentioned that, due to the large aspect ratio of this flow problem, the crossstreamwise direction in the corresponding contour plots is stretched by a factor of 2 .

Inspection of the contour plots in Figures 1 and 2 indicates that, velocities of helium and xenon are almost identical. This implies that for the cases I and II, the components have relaxed toward a common thermodynamic equilibrium. Discrepancies are, however, remarkable for case III (see Figure 3) in the sense that the constituents flow with distinct velocities. This occurs since under such a rarefied condition, the frequency of molecular collision is too low that prohibits a local thermodynamic equilibrium between the component species.

In what concerns the distribution of streamwise velocity, the multifluid results at about $4 / 5$ of the channels' length from the inlet are compared with those of the Navier-Stokes equations in Figure 4. The presented results correspond to the three cases of the gas rarefaction and have been normalized with centerline velocities at that section (i.e., $U_{c}$ ).

Inspection of Figure 4(a) indicates that, under low rarefaction, the multifluid results correspond well with those of the Navier-Stokes equations. Obviously, the constituents of the mixture reach the same thermodynamic equilibrium. Equilibrium also establishes between the walls and the mixture since velocity-slip is vanishing. Such a behavior allows one to conclude that under low rarefaction, the current multifluid description automatically goes back to the Navier-Stokes equations with no-slip/no-temperature jump boundary conditions.

It can be witnessed in Figure 4(b) that under moderate rarefaction (i.e., case II) velocity-slip initiates to appear. This implies that the mixture is not in complete equilibrium with the walls. Meanwhile, thermodynamic equilibrium between the constituents ceases to establish in the vicinity of the walls since some discrepancies occur in the helium and xenon velocities there. Consequently, it is not surprising to see that the results of the multifluid description differ from those of the Navier-Stokes equations in near wall regions. 
Journal of Thermal Engineering, Research Article, Vol. 6, No. 3, pp. 405-421, April, 2020

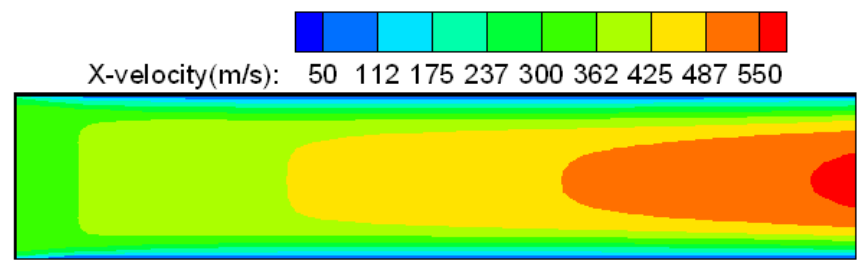

(a) Helium

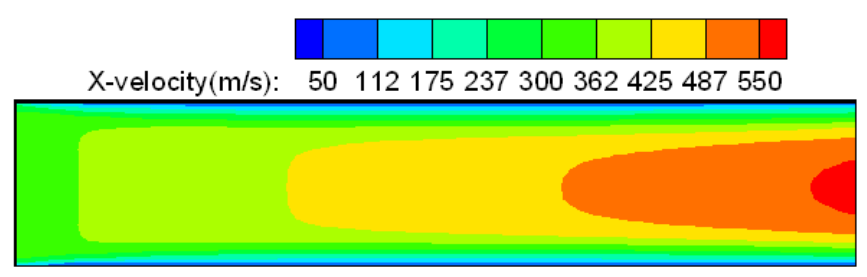

(b) Xenon

Figure 1. X-velocity contours for the mixture constituents in case $\mathrm{I}(K n \approx 0.001)$ of the He-Xe mixture

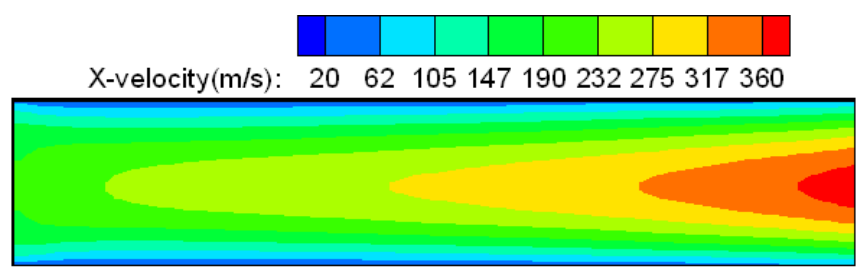

(a) Helium

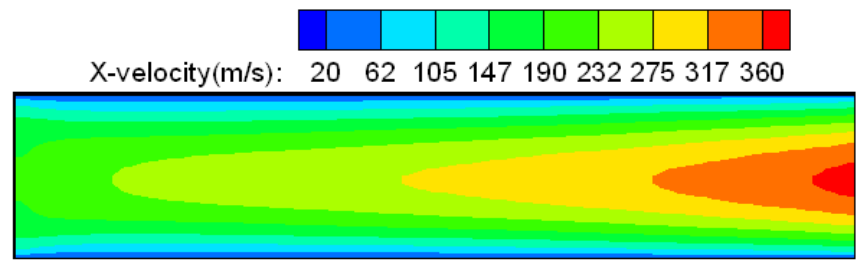

(b) Xenon

Figure $2 . \mathrm{X}$-velocity contours for the mixture constituentsin case II $(K n \approx 0.01)$ of the $\mathrm{He}-\mathrm{Xe}$ mixture

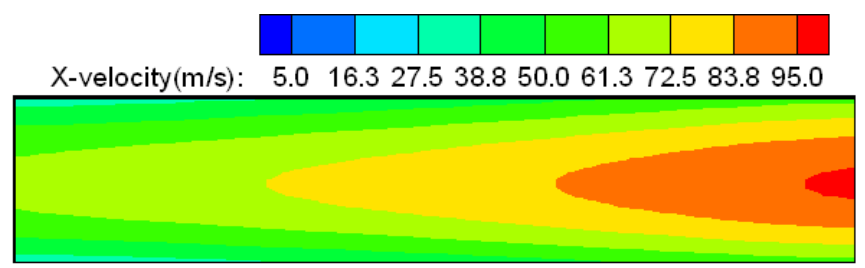

(a) Helium

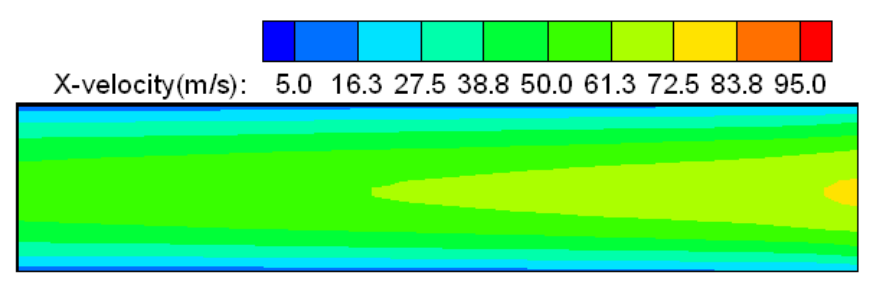

(b) Xenon

Figure 3. $\mathrm{X}$-velocity contours for the mixture constituents in case III $(K n \approx 0.1)$ of the $\mathrm{He}-\mathrm{Xe}$ mixture 


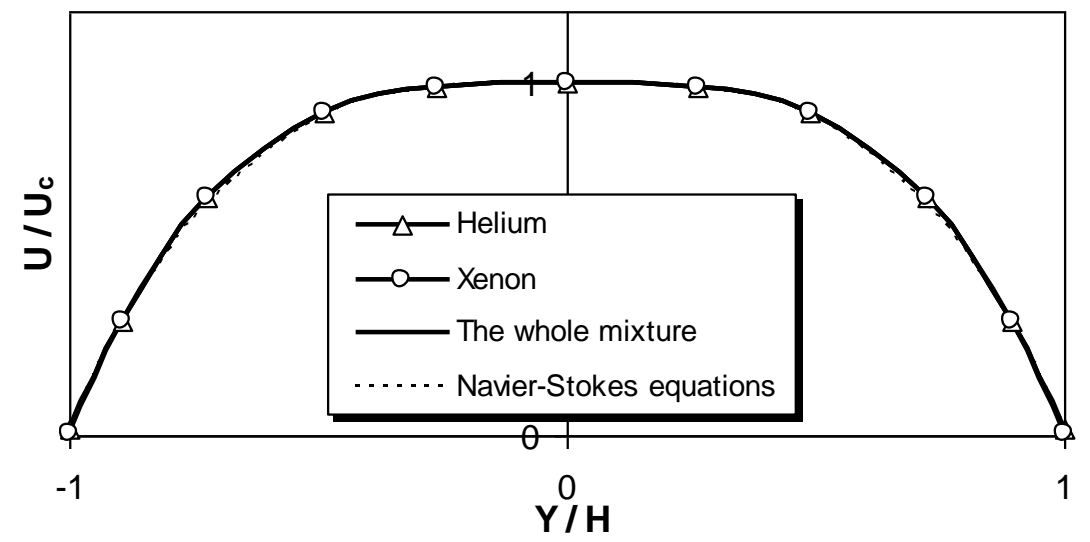

(a) Case I $(K n \approx 0.001)$

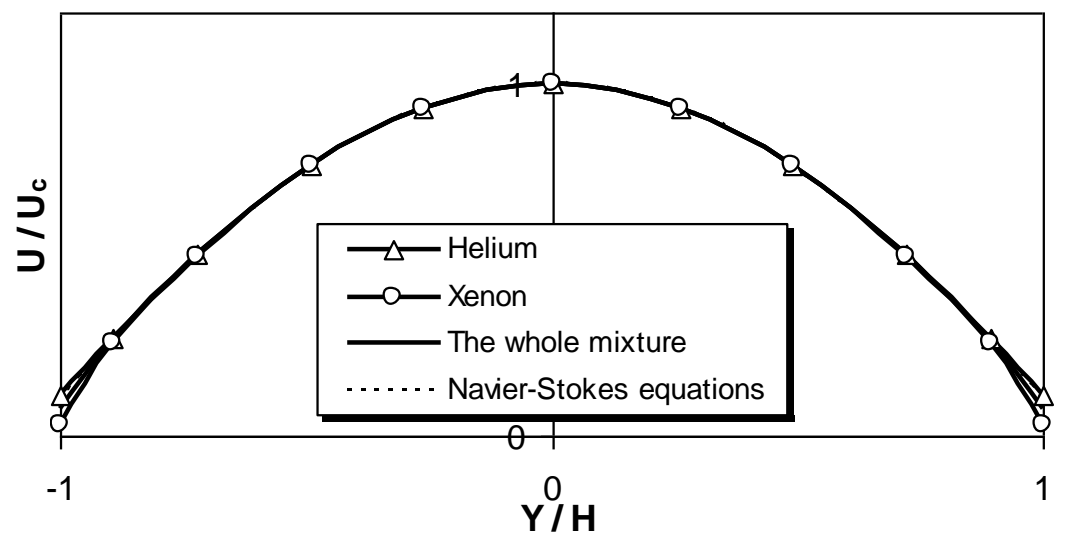

(b) Case II $(K n \approx 0.01)$

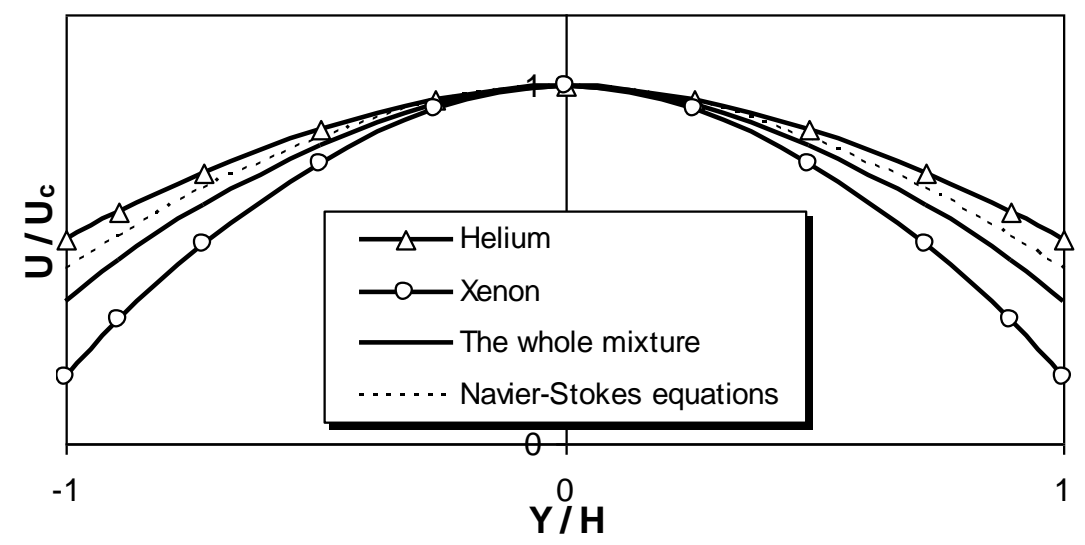

(c) Case III $(K n \approx 0.1)$

Figure 4. Velocity profiles at about $4 / 5$ of the channels' length from the inlet in the $\mathrm{He}-\mathrm{Xe}$ mixtures

Indeed, the Navier-Stokes equations are based on the concept of local equilibrium between the constituents and thereby they cannot properly explain the non-equilibrium effects that appear between the mixture constituents under rarefied conditions. Figure 4(c) illustrates that for the highly rarefied flow of case III, velocities of the constituents are completely distinct and the mixture exhibits strong non-equilibrium effects over the entire flow 
region. Consequently, the Navier-Stokes equations lose their accuracy here and the flow field can only be described by the developed multifluid model. Such a behavior strongly supports the superiority of the current multifluid description for the study of non-equilibrium rarefied flows. Consequently, in what follows, the description will be utilized for some analysis of the rarefied gas mixtures.

\section{Variations of Slip Velocities Along the Plates}

Variations of slip velocities of the mixtures constituents along the plates are analyzed in this section. For this purpose, velocity distributions of helium and xenon in the vicinity of the plates are plotted in Figure 5. The presented results correspond to the three cases of the gas rarefaction. It must be noted that, velocity magnitudes for each case have been normalized with the inlet centerline velocity of the whole mixture (i.e., $U_{i}$ ).

The figure demonstrates that, findings of Figure 4 can be easily generalized to the entire plates. Indeed, the slip velocities are vanishingly small at $K n \approx 0.001$ and the constituents relax towards a common thermodynamic equilibrium at the walls. Such equilibrium, however, ceases to establish as the degree of gas rarefaction increases. A closer scrutiny of the figure indicates that, slip velocity of the lighter species is always higher.

Under these circumstances, densities of the mixtures constituents are almost identical. Consequently, it is not surprising to see that for each case, the whole mixture flows with a velocity that is nearly average of those of helium and xenon. Note thatv $=\left(\rho_{H e} \mathbf{v}_{H e}+\rho_{X e} \mathbf{v}_{X e}\right) /\left(\rho_{H e}+\rho_{X e}\right)$.

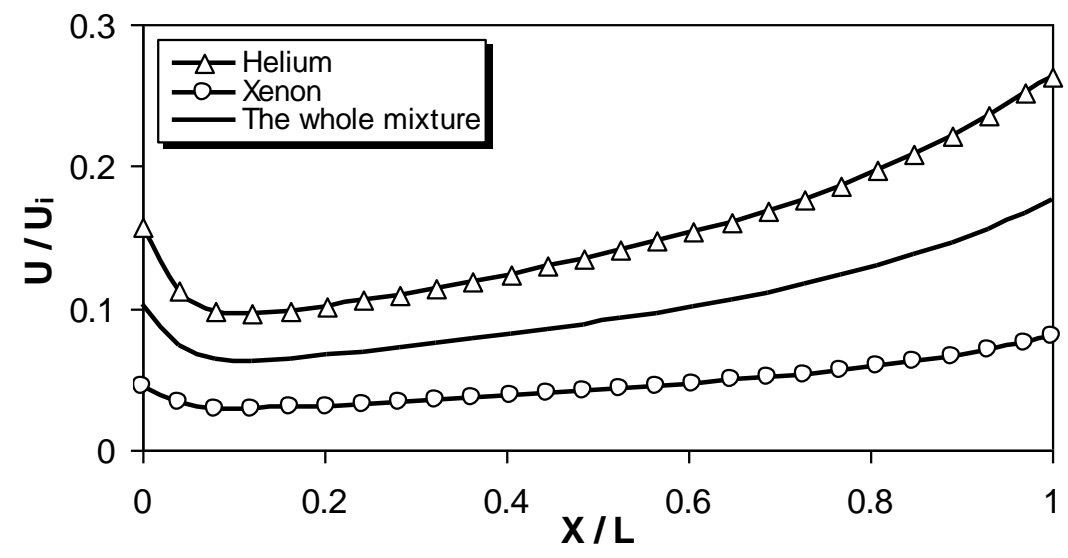

(a) Case II $(K n \approx 0.01)$

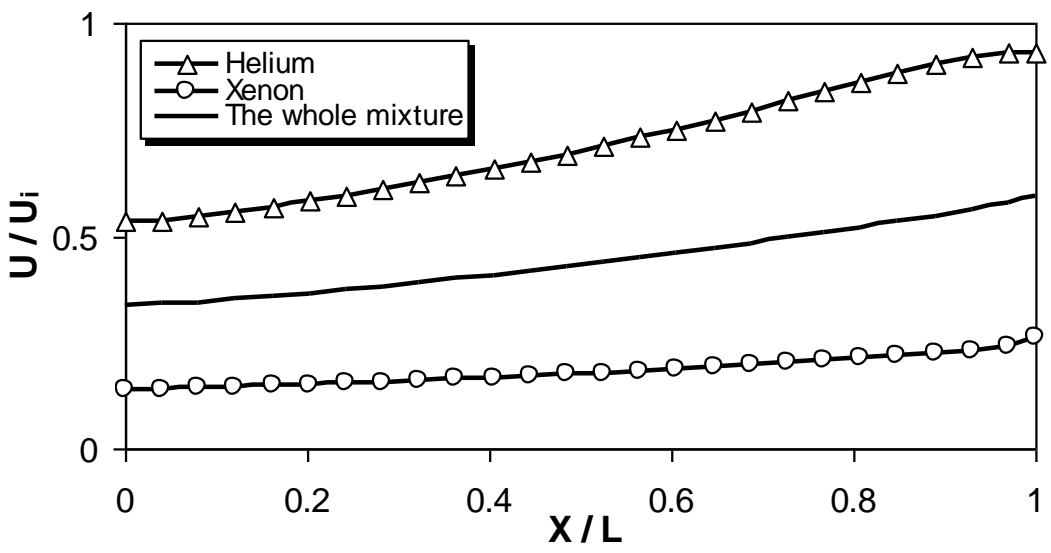

(b) Case III $(K n \approx 0.1)$

Figure 5. Velocity distribution in the vicinity of the platesfor the $\mathrm{He}-\mathrm{Xe}$ mixtures 


\section{Importance of Velocity-Slip}

One reason accounting for the appearance of non-equilibrium features between component species of a rarefied gas mixture is attributed to the use of separate velocity-slip and temperature-jump boundary conditions for each of the constituents. To clarify how this effect may influence the degree of non-equilibrium in the current flow problem, computations of case III (namely, $K n \approx 0.1$ ) are repeated here for an artificial case wherein the no-slip condition is applied to the walls.

Figure 6 depicts $\mathrm{x}$-velocity contours of the mixture constituents for such a simulation. It can be witnessed that the discrepancies are not vanishing even in the absence of velocity-slip at the walls. This allows one to conclude that, non-equilibrium features appearing between the mixtures constituents are not solely due to the application of separate boundary conditions.

Comparing the contour plots in Figures 6 and 3 illustrates the influence of gas slip on the establishment of the velocity fields. Evidently, by including velocity-slip, the constituents flow faster not only in the vicinity of the walls but also in the core flow region. Such a behavior is more obviously explained in Figure 7 where the distributions of streamwise velocities at about $4 / 5$ of the channel's length from the inlet are provided.

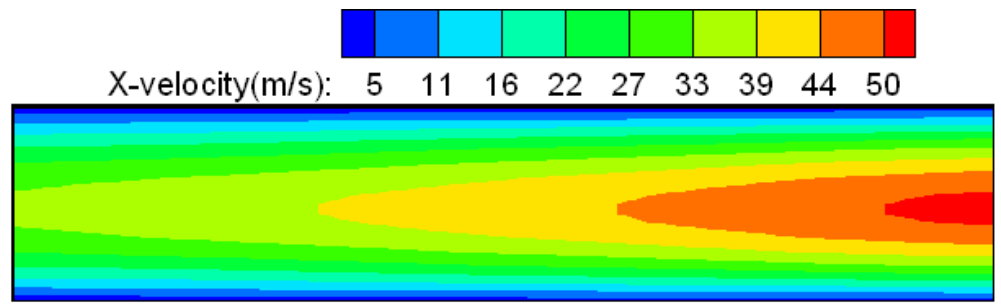

(a) Helium

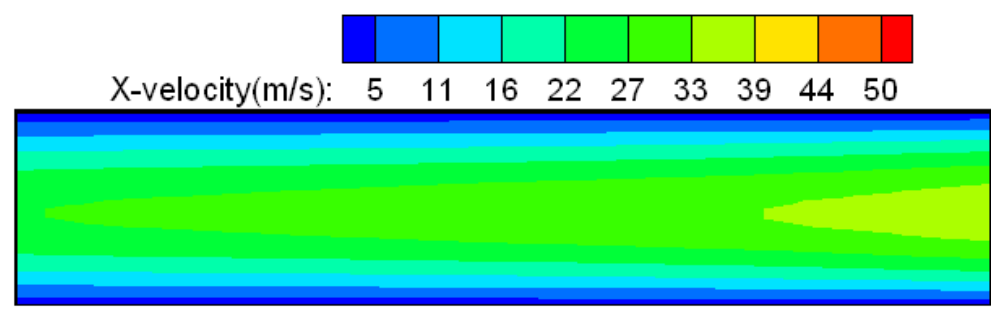

(b) Xenon

Figure 6. $\mathrm{X}$-velocity contours for the mixture constituents at $K n \approx 0.1$ in the absence of velocity-slip at the walls

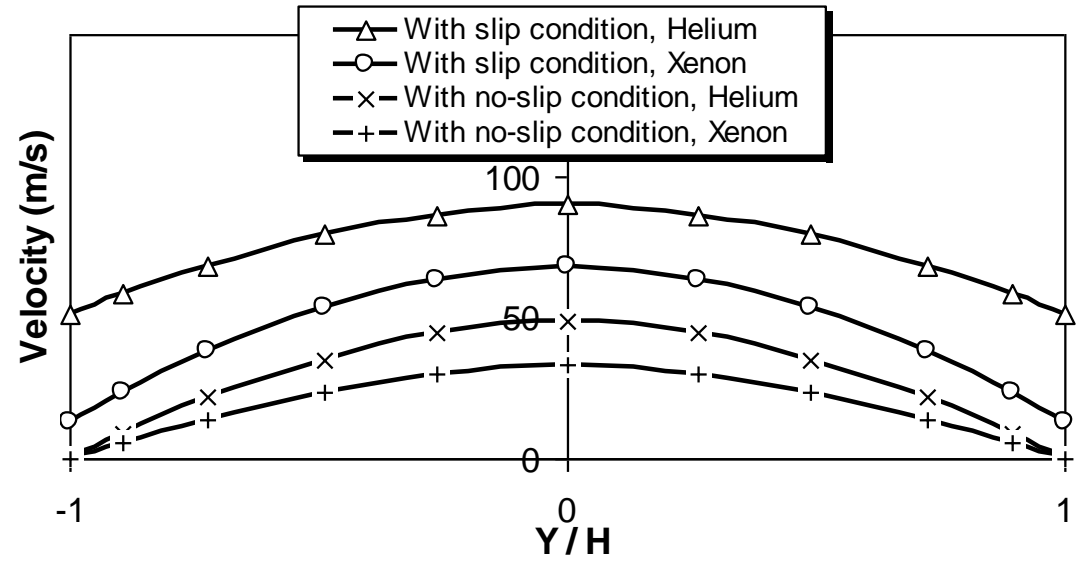

Figure 7. Velocity profiles at about $4 / 5$ of the channel's lengthfrom the inlet in the $\mathrm{He}-\mathrm{Xe}$ mixture at $K n \approx 0.1$ 
Journal of Thermal Engineering, Research Article, Vol. 6, No. 3, pp. 405-421, April, 2020

\section{Effect of Tangential-Momentum-Accomodation Coefficient}

This section is devoted to clarify the effect of tangential-momentum-accommodation coefficient $(\sigma)$ on the degree of non-equilibrium between component species of a rarefied gas mixture. To this aim, computations at $K n \approx$ 0.1 are repeated here for parallel plates with $\sigma=0.1, \sigma=0.5$, and $\sigma=1$ and the corresponding results are compared. The comparison is first made in Figures 8-10 where $\mathrm{x}$-velocity contours of the mixtures constituents are displayed. Notice that as the walls approach a specular reflector (i.e., $\sigma \rightarrow 0$ ), velocity magnitudes increase over the entire flow field. To demonstrate this effect further, Figure 11 plots the streamwise velocity profiles at about $4 / 5$ of the channel's length from the inlet for the three accommodation coefficients. The figure indicates that with decrease in the accommodation coefficient, non-equilibrium features between the mixtures constituents become more pronounced especially in near wall regions.

\section{Influence of Mass Disparity of the Mixture Constituents}

Simulation results of the current flow problem led to the conclusion that as the overall Knudsen number approaches 0.1 , considerable discrepancies appear between the mixture constituents. This occurs since molecular masses of helium and xenon are quite distinct. Note that $m_{X e} / m_{H e} \approx 32$.

To demonstrate how mass disparity of the mixture constituents may influence the degree of nonequilibrium, multifluid computations at $K n \approx 0.1$ are repeated here for $\mathrm{He}-\mathrm{Ne}, \mathrm{He}-\mathrm{Ar}$, and $\mathrm{He}-\mathrm{Kr}$ mixtures and the corresponding results are compared. The ratios of the molecular masses for these mixtures are $m_{\mathrm{Ne}} / m_{\mathrm{He}} \approx 5$, $m_{\mathrm{Ar}} / m_{\mathrm{He}} \approx 10$, and $m_{\mathrm{Kr}} / m_{\mathrm{He}} \approx 21$, respectively. As a consequence, the mixtures exhibit distinct mass disparities. Figures 12-14 plot $\mathrm{x}$-velocity contours for the constituents of these mixtures.

In a general way notice that as the molecular mass ratio increases, velocities of the constituents become more distinct. This indicates that, in addition to overall Knudsen number, the mass disparity of the mixture constituents may influence the degree of non-equilibrium between component species of a rarefied gas mixture. A closer scrutiny of the figures demonstrates that, in the circumstance of non-equilibrium, the lighter species flows with higher velocities over the entire flow field. It is also noticed that velocities of the constituents and thereby the mixture velocity is lower for heavier mixtures, as may be expected.

Inspection of the previously presented simulation results led to the conclusion that the Navier-Stokes equations lose their accuracy under rarefied conditions. This occurred as a consequence of the existence of nonequilibrium effects between component species of rarefied gas mixtures. In spite of this, it has been found that when molecular masses of the constituents are not quite distinct, the appearance of non-equilibrium features will be delayed. Concerning this, one may conclude that when the constituents are almost identical it is possible for the Navier-Stokes equations to still remain true in rarefied circumstances.

\section{CONCLUSION}

In this study, multifluid balance equations in conjunction with proposed rarefied boundary conditions for velocity-slip and temperature-jump were solved for some microchannel flow problems. Simulation results are compared with those of a Navier-Stokes solver. Based on the presented results, the following conclusions may be drawn:

- The proposed multifluid description offers an effective simulation method for the study of gas mixture flows under rarefied conditions.

- When the degree of gas rarefaction is low, the current multifluid description automatically goes back to the Navier-Stokes equations with no-slip/no-temperature jump boundary conditions.

- While the Navier-Stokes equations may lose their accuracy under high rarefaction, non-equilibrium features are properly captured by the proposed multifluid description.

During the current simulations, arbitrary conditions were used. The developed solution procedure is, however, applicable to a wide range of applications for monoatomic gas mixtures. It is noteworthy that since the current multifluid model relies on $5 v$-moment theory, it is applicable to the slip flow regime (i.e., when $0.001<$ 
$K n<0.1$ ). But, the model cannot be accurate in the transition flow regime (i.e., when $K n>0.1$ ) because constitutive relations cease to be linear there.

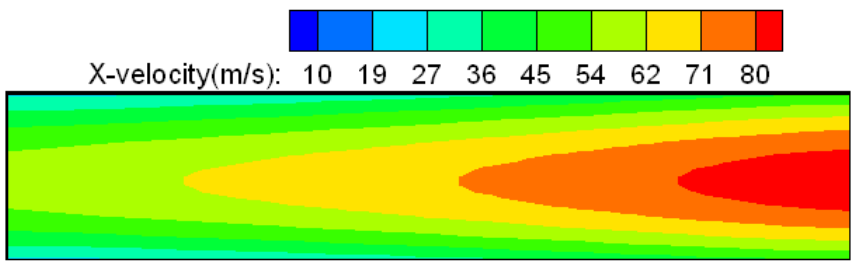

(a) Helium

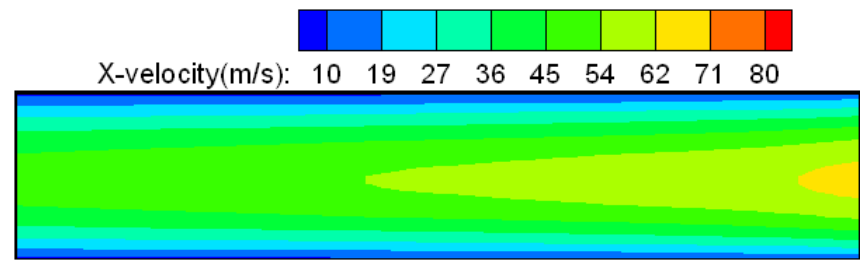

(b) Xenon

Figure 8. $\mathrm{X}$-velocity contours of the mixture constituentsat $K n \approx 0.1$ with $\sigma=1$

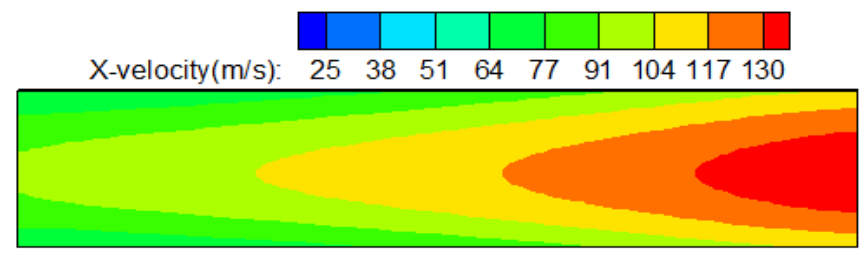

(a) Helium

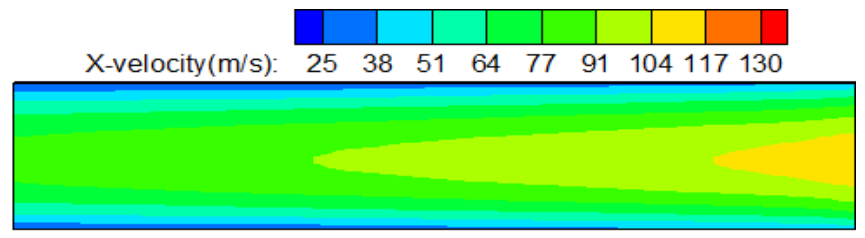

(b) Xenon

Figure 9. $\mathrm{X}-$-velocity contours of the mixture constituentsat $K n \approx 0.1$ with $\sigma=0.5$

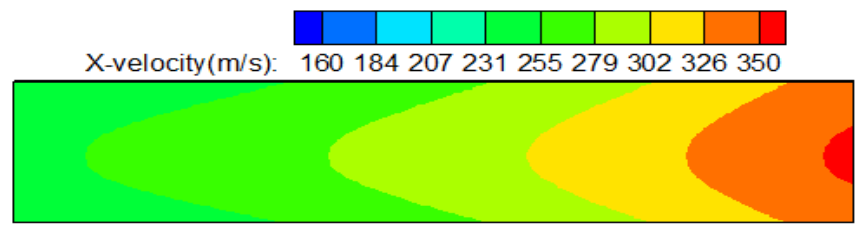

(a) Helium

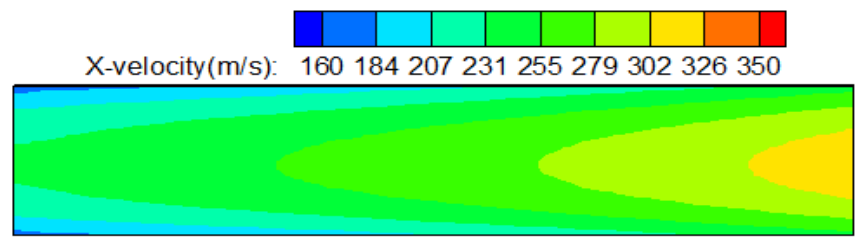

(b) Xenon

Figure 10. $\mathrm{X}$-velocity contours of the mixture constituentsat $K n \approx 0.1$ with $\sigma=0.1$ 
Journal of Thermal Engineering, Research Article, Vol. 6, No. 3, pp. 405-421, April, 2020

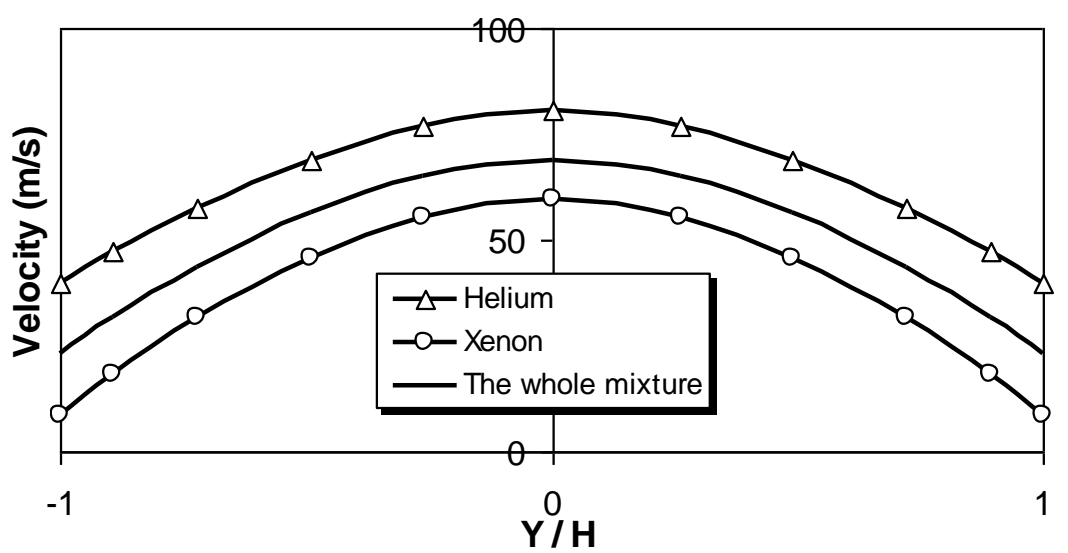

(a) $\sigma=1$

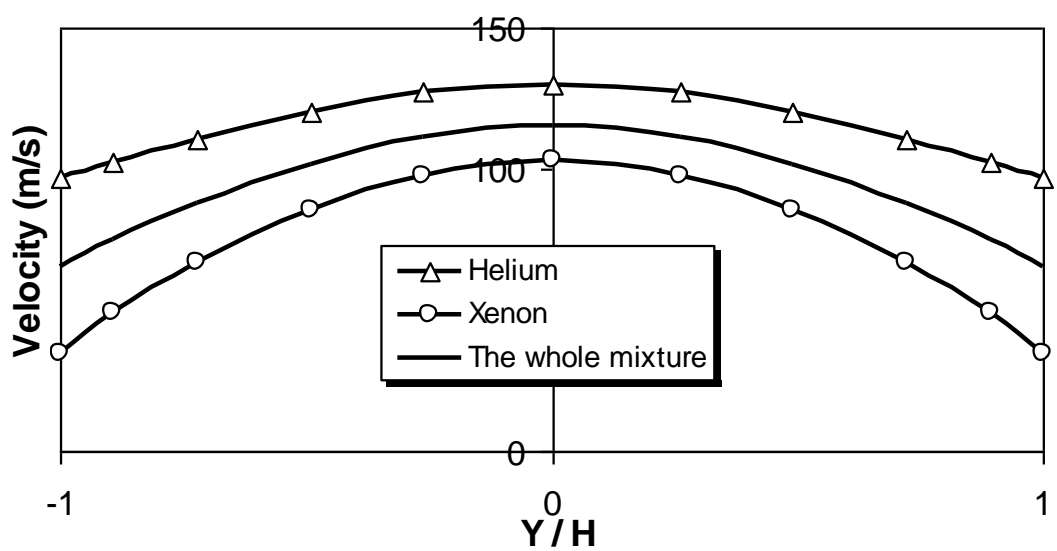

(b) $\sigma=0.5$

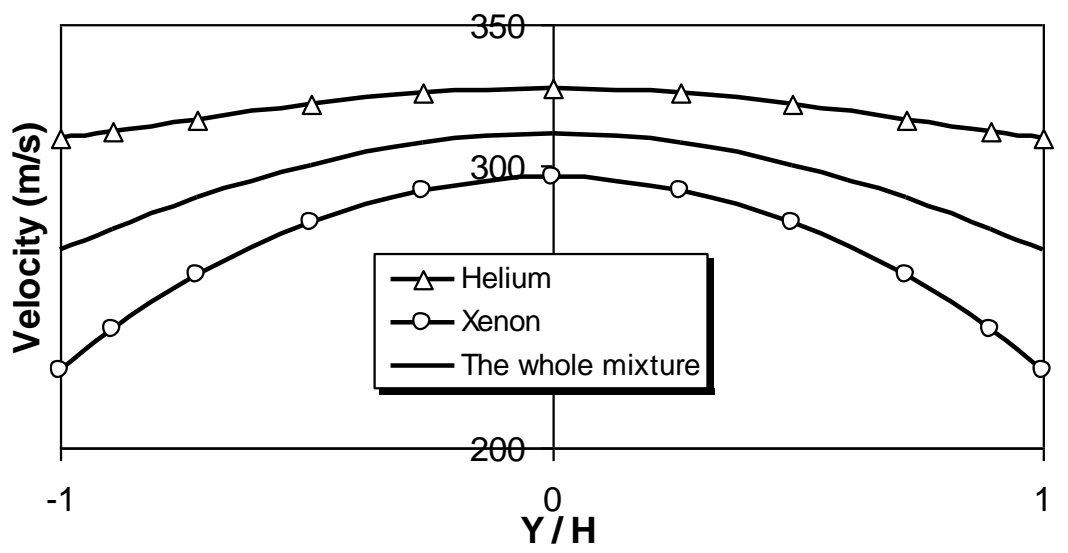

(c) $\sigma=0.1$

Figure 11. Velocity profiles at about $4 / 5$ of the channel's length from the inlet in the He-Xe mixtures at $K n \approx 0.1$ 
Journal of Thermal Engineering, Research Article, Vol. 6, No. 3, pp. 405-421, April, 2020

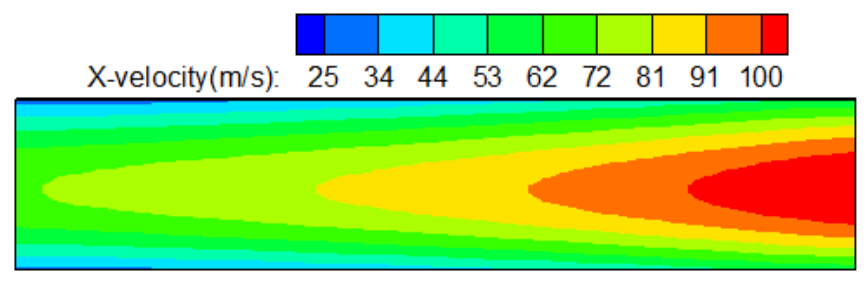

(a) Helium

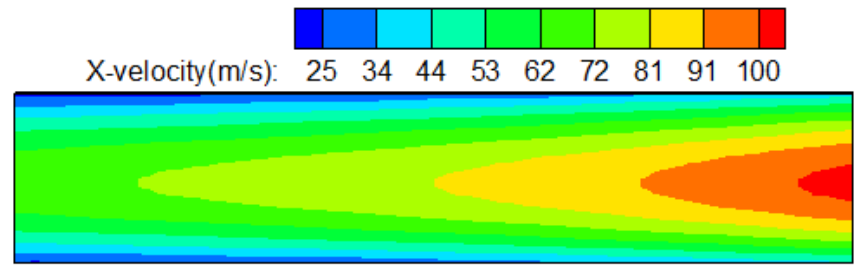

(b) Neon

Figure 12. $\mathrm{X}$-velocity contours for the constituents of the He-Ne mixture at $K n \approx 0.1$

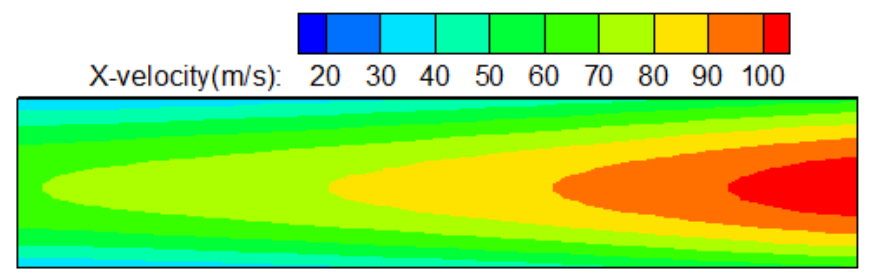

(a) Helium

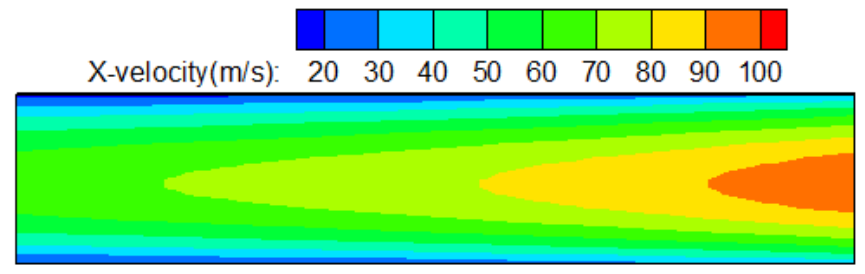

(b) Argon

Figure 13. $\mathrm{X}$-velocity contours for the constituents of the $\mathrm{He}-\mathrm{Ar}$ mixture at $K n \approx 0.1$

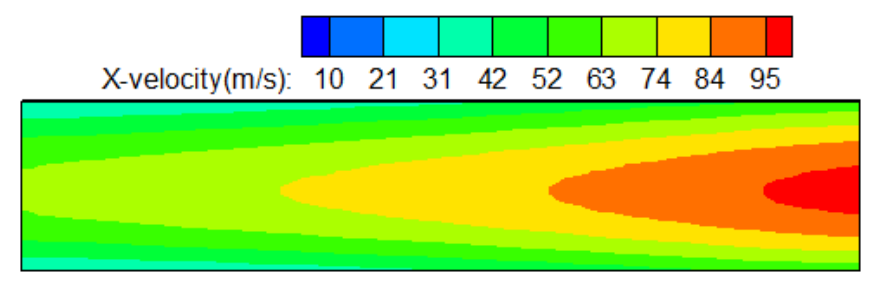

(a) Helium

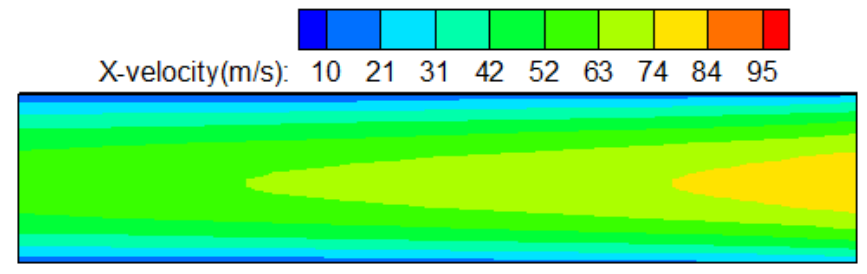

(a) Krypton

Figure 14. $\mathrm{X}$-velocity contours for the constituents of the $\mathrm{He}-\mathrm{Kr}$ mixture at $\mathrm{Kn} \approx 0.1$ 


\section{NOMENCLATURE}

$c_{i}$

$E$

$\tilde{E}$

$H$

Kn

$L$

$m$

$m_{\alpha \beta}$

$M_{\alpha \beta}$

$M_{\beta \alpha}$

$n$

$p$

$p_{e}$

$p_{t, i}$

$P r$

$q$

$L$

$R$

$t$

$T$

$T_{t, i}$

$\tilde{T}$

$u, v$

V

$\tilde{\mathbf{v}}$

$x, y$

Greek symbols

$\gamma$

$\eta_{\alpha \alpha}$

$\eta_{\alpha \beta}$

$\eta_{\beta \alpha}$

$\eta_{\beta \beta}$

$\lambda$

$\lambda_{\alpha}$

$\lambda_{\beta}$

$\lambda_{\alpha \alpha}$

$\lambda_{\alpha \beta}$

$\lambda_{\beta \alpha}$

$\lambda_{\beta \beta}$

$\rho$

$\sigma$

$\sigma_{T}$

$\boldsymbol{\tau}_{i j}$

$v_{\alpha \beta}$

$v_{\beta \alpha}$

$\boldsymbol{\Omega}_{\alpha \beta}^{(i, j)}$

\section{Subscripts}

S

$t$ inlet mixture concentration

total energy per unit volume

collision parameter

half of the distance between the plates

overall Knudsen number of the whole mixture

length of the channel

molecular mass

reduced mass of the gas mixture

transport coefficient associated with thermal-diffusion effects

transport coefficient associated with thermal-diffusion effects

normal direction

pressure

exit pressure

inlet stagnation pressure

Prandtl number

heat flux vector

length of the channel

gas constant

time

temperature

inlet stagnation temperature

collision parameter

components of velocity vector

velocity vector

collision parameter

Cartesian coordinates

ratio of the specific heats

transport coefficient associated with viscous effects transport coefficient associated with viscous effects transport coefficient associated with viscous effects transport coefficient associated with viscous effects overall mean-free-path of the whole mixture mean-free-path of the constituent $\alpha$ in the mixture mean-free-path of the constituent $\beta$ in the mixture transport coefficient associated with thermal effects transport coefficient associated with thermal effects transport coefficient associated with thermal effects transport coefficient associated with thermal effects mass density tangential-momentum-accommodation coefficient thermal-accommodation coefficient viscous stress tensor collision frequency for cross-collision between species $\alpha$ and $\beta$ molecules collision frequency for cross-collision between species $\beta$ and $\alpha$ molecules Chapman-Cowling collision integrals

slip

tangential 
Journal of Thermal Engineering, Research Article, Vol. 6, No. 3, pp. 405-421, April, 2020

wall

$\alpha, \beta \quad$ mixture constituents

\section{REFERENCES}

[1] Goli A, Zahmatkesh I. Slip flow in porous micro-tubes under local thermal non-equilibrium conditions. Transp Phenom Nano Micro Scales 2018;6:79-87. doi:10.22111/TPNMS.2018.4034.

[2] Yan F, Farouk B. Numerical simulation of gas flow and mixing in a microchannel using the direct simulation Monte Carlo method. Microscale Thermophys Eng 2002;6:235-251. doi:10.1080/10893950290098296.

[3] Wang M, Li Z. Gas mixing in microchannels using the direct simulation Monte Carlo method. Int J Heat Mass Transf 2006;49:1696-1702. doi:10.1016/j.ijheatmasstransfer.2005.10.022.

[4] Hosseinalipour SM, Jabbari E, Madadelahi M, Fardad A. Gas mixing simulation in a T-Shape micro channel using the DSMC method. Transp Phenom Nano Micro Scales 2014;2:132-139. doi:10.7508/TPNMS.2014.02.005.

[5] Amini Y, Emdad H, Akramian K, Bordbar F. Investigation of the common nose cone shapes in different gas mixtures in high Knudsen numbers. Scientia Iranica B 2012;19:1511-1518. doi:10.1016/j.scient.2012.10.029.

[6] Vargas M, Stefanov S, Roussinov V. Transient heat transfer flow through a binary gaseous mixture confined between coaxial cylinders. Int J Heat Mass Transf 2013;59:302-315. doi:10.1016/j.ijheatmasstransfer.2012.12.025.

[7] Naris S, Valougeorgis D, Kalempa D, Sharipov F. Gaseous mixture flow between two parallel plates in the whole range of the gas rarefaction. Physica A 2004;336:294-311. doi:10.1016/j.physa.2003.12.047.

[8] Kosuge S, Takata S. Database for flows of binary gas mixtures through a plane microchannel. Eur J Mech B Fluids 2008;27:444-465. doi:10.1016/j.euromechflu.2007.08.002.

[9] Naris S, Valougeorgis D, Kalempa D, Sharipov F. Flow of gaseous mixtures through rectangular microchannels driven by pressure, temperature, and concentration gradients. Phys Fluids 2005;17:100607. doi:10.1063/1.1896986.

[10] McCormack FJ. Construction of linearized kinetic models for gaseous mixtures and molecular gases. Phys Fluids 1973;16:2095-2105. doi:10.1063/1.1694272.

[11] Szalmas L, Valougeorgis D. Rarefied gas flow of binary mixtures through long channels with triangular and trapezoidal cross sections. Microfluid Nanofluid 2010;9:471-487. doi:10.1007/s10404-010-0564-9.

[12] Polikardiv AP, Ho MT, Graur I. Transient heat transfer in a rarefied binary gas mixture confined between parallel plates due to a sudden small change of wall temperatures. Int J Heat Mass Transf 2016;101:1292-1303. doi:10.1016/j.ijheatmasstransfer.2016.05.124.

[13] Ho MT, Wu L, Graur I, Zhang Y, Reese JM. Comparative study of the Boltzmann and McCormack equations for Couette and Fourier flows of binary gaseous mixtures. Int J Heat Mass Transf 2016;96:29-41. doi:10.1016/j.ijheatmasstransfer.2015.12.068.

[14] Tantos C, Valougeorgis D. Conductive heat transfer in rarefied binary gas mixtures confined between parallel plates based on kinetic modeling. Int J Heat Mass Transf 2018;117:846-860. doi:j.ijheatmasstransfer.2017.10.050.

[15] Kosuge S. Model Boltzmann equation for gas mixtures: Construction and numerical comparison. Eur J Mech B Fluids 2009;28:170-184. doi:10.1016/j.euromechflu.2008.05.001.

[16] Tantos C. Steady planar Couette flow of rarefied binary gaseous mixture based on kinetic modeling. Eur J Mech B Fluids 2019;76: 375-389. doi:10.1016/j.euromechflu.2019.04.005.

[17] Yamaguchi H, Hosoi J, Matsuda Y, Niimi T. Measurement of conductive heat transfer through rarefied binary gas mixtures. Vacuum 2019;160:164-170. doi:10.1016/j.vacuum.2018.11.021.

[18] Zahmatkesh I, Alishahi MM, Emdad H. New velocity-slip and temperature-jump boundary conditions for Navier-Stokes computation of gas mixture flows in microgeometries. Mech Res Commun 2011;38:417-424. doi:10.1016/j.mechrescom.2011.06.001.

[19] Zahmatkesh I, Emdad H, Alishahi MM. Navier-Stokes computation of some gas mixture problems in the slip flow regime. Scientia Iranica B 2015;22:187-195. 
[20] Crouzet F, Daude F, Galon P, Hérard JM, Hurisse O, Liu Y. Validation of a two-fluid model on unsteady watervapour flows. Comput Fluids 2015;119:131-142. doi:10.1016/j.compfluid.2015.06.035.

[21] Torshizi E, Zahmatkesh I. Comparison between single-phase, two-phase mixture and Eulerian-Eulerian models for the description of jet impingement of nanofluids. J Appl Comput Sci Mech 2016;27:55-70. doi:10.22067/fum_mech.v27i2.41797.

[22] Zahmatkesh I, Torshizi E. Scrutiny of unsteady flow and heat transfer in a backward-facing step under pulsating nanofluid blowing using the Eulerian-Eulerian approach. J Mech 2019;35:93-105. doi:10.1017/jmech.2017.73.

[23] Fox RO. A kinetic-based hyperbolic two-fluid model for binary hard-sphere mixtures. J Fluid Mech 2019;877:282-329. doi:10.1017/jfm.2019.608.

[24] Shin HC, Seo HS, Kim SM. Two-dimensional two-fluid model for air-oil wavy flow in horizontal tube. J Mech Sci Technol 2019;33:2693-2709. doi:10.1007/s12206-019-0517-5.

[25] Zahmatkesh I, Emdad H, Alishahi MM. Importance of molecular interaction description on the hydrodynamics of gas mixtures. Scientia Iranica B 2011;18:1287-1296. doi:10.1016/j.scient.2011.08.032.

[26] Zahmatkesh I, Emdad H, Alishahi MM. Two-fluid analysis of a gas mixing problem. Scientia Iranica B 2013;20:162-171. doi:10.1016/j.scient.2012.12.017.

[27] Zahmatkesh I, Emdad H, Alishahi MM. Viscous and inviscid solutions of some gas mixture problems. Heat Transf Res 2011;42:233-250. doi: 10.1615/HeatTransRes.2011002769.

[28] Zahmatkesh I, Emdad H, Alishahi MM. Effect of temperature level on parallel mixing of two gas streams. Mech Res Commun 2011;38:141-145. doi:10.1016/j.mechrescom.2011.01.004.

[29] Maxwell JC. On stresses in rarefied gases arising from inequalities of temperature. Philos Trans R Soc London, Ser B 1879;170:231-256. doi:10.1098/rstl.1879.0067.

[30] Smoluchowski M. On conduction of heat by rarefied gases (Ueber warmeleitung in verdunnten gasen). Ann Phys Chem 1898;64:101-130. doi:10.1002/andp.18983000110.

[31] Roe PL. Discrete models for numerical analysis of time-dependent multidimensional gas dynamics. J Comput Phys 1986;63:458-476. doi:10.1016/0021-9991(86)90204-4. 